\title{
Uniform Segregation of Densely Deployed Wireless Sensor Networks
}

\author{
Manjeet Singh, Surender Soni \\ ECE Department \\ NIT Hamirpur \\ Hamirpur, H.P., India
}

\begin{abstract}
In wireless sensor networks, the selection of cluster heads relies upon the various selection parameters, such as energy, distance, node concentration and rate of retransmission. There is always uncertainty in the suitability of sensor node for the cluster head role due to these various selection parameters. Fuzzy logic is capable of overcoming uncertainties even with incomplete available information. This quality of fuzzy logic can reduce uncertainty in cluster head selection up to large extent. Therefore, in this paper, a fuzzy logic based clustering approach is proposed to enhance the network operational lifetime. The cluster formation is done on the basis of the spatial correlation value between sensors to organize clusters uniformly in the network. The results are compared with well-known approaches CHEF and LEACH.
\end{abstract}

Keywords-Clustering; fuzzy logic; wireless sensor network; cluster head; uncertainty

\section{INTRODUCTION}

The recent research and advancement in wireless sensor networks (WSNs) are paving the way in making the world smarter in term of technology to control and monitor various activities remotely. The WSNs are special kind of network and their dependency on battery lifetime is the major problem. The design objective of every approach is to extend the lifetime of the WSN by saving battery power without jeopardizing its overall quality of service. The most favored approach to utilizing battery power more efficiently is dividing the whole network into clusters and allow only cluster heads to communicate with the base station (BS) as shown in Fig. 1.

The clustering can be static or dynamic. The authors in [1] investigated the impact of static and dynamic clustering on network lifetime and claim that the dynamic clustering is more efficient. The dynamic clustering brings new challenges like suitable cluster head selection, cluster heads rotation and uniformity of clusters in the network. Many authors [2]-[4] are inspired by low energy adaptive clustering hierarchy (LEACH) [1] and they investigated the shortcoming and come up with new solutions. They accounted a number of parameters in their selection process but still due to uncertainty in decision making some time inefficient cluster heads gets selected and degrade overall performance.

The inefficient cluster head cannot maximize the network lifetime. Therefore, authors in [5] utilized fuzzy logic to deal with uncertainty in cluster head selection. However, the proposed approach was centralized and have limited applications. To overcome limitations of the centralized approach the authors presented localized clustering technique by clubbing LEACH [1] and fuzzy logic together [6]. The main problem in cluster head election mechanism using fuzzy logic (CHEF) [6] is that its cluster heads selection depends upon the random number and due to optimal cluster radius cluster heads can reduce from below optimal value.

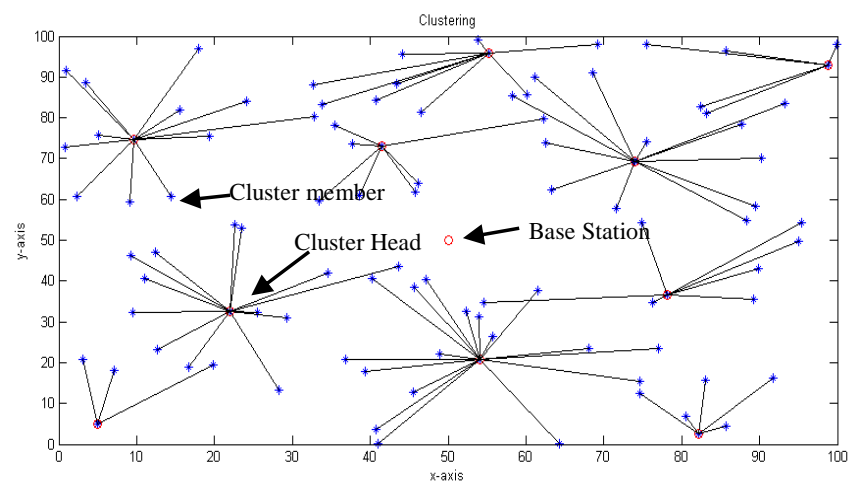

Fig. 1. The clustered architecture of WSN.

The inefficient cluster head cannot maximize the network lifetime. Therefore, authors in [5] utilized fuzzy logic to deal with uncertainty in cluster head selection. However, the proposed approach was centralized and have limited applications. To overcome limitations of the centralized approach the authors presented localized clustering technique by clubbing $\mathrm{LEACH}$ [1] and fuzzy logic together [6]. The main problem in CHEF [6] is that its cluster heads selection depends upon the random number and due to optimal cluster radius cluster heads can reduce from below optimal value.

In [7]-[10] authors employed the basic architecture of Gupta's approach [5] with different input descriptors. The network lifetime can extend by using other fuzzy inference systems rather than Mamdani model is shown in [11]. Some authors [12]-[15] take the advantage of fuzzy type-2 system in order to further reduce uncertainty in cluster head election. It is seen that the approaches used type- 1 fuzzy system perform better as compared the approaches used the type-2 fuzzy system. The neural network applications in WSN are addressed in [16]. The cluster head failure issue has been addressed in [17], [18] and introduced the backup cluster heads (BCHs) concept based upon fuzzy logic output value. The heterogeneity in the network is exploited using fuzzy logic in [19]. The segregation of network into tiers based on distance has been done in [20]. The effects of various 
parameters on network lifetime have been analyzed and presented in [21]. The researchers developed various approaches to cover different issues arises during the clustering process. However, the spatial correlation has not been exploited to form uniform clusters in the network and to define optimal cluster radius [22]. Therefore, in this paper, a fuzzy logic based novel clustering is presented where cluster heads election depends upon the fuzzy system output value and cluster formation depends upon the spatial correlation value between the sensor nodes to achieve the objective of prolonging network lifetime.

The rest of the paper is structured as follows: In Section 2 related work is reviewed to identify the research gaps. The problem is formulated in Section 3. The proposed approach is developed and discussed in Section 4. The results are presented in Section 5 and the paper is concluded in Section 6.

\section{RELATED WORK}

In this section, fuzzy based clustering techniques in WSNs are briefly reviewed. In [5] authors have been investigated the limitations of LEACH [1] protocol and further proposed novel fuzzy-based clustering techniques based on the finding. It is a centralized clustering scheme, where three input variables: node concentration, energy level, and centrality are used to evaluate the chance value of sensor nodes. The base station is responsible for distributing cluster heads uniformly in the network. Gupta's technique has some limitations like centralized clustering, location awareness required, not explained the meaning of medium size clusters in term of a number of nodes.

Researchers in [6], highlighted the limitations of the [5] approach and further presented CHEF. In CHEF decision related to cluster head selection is taken at node level rather than at centralized level. Energy and local distance are used as fuzzy input variables to evaluate the chance value of sensor nodes. This technique is more complex as compared to LEACH because it performs LEACH operation first and then used fuzzy rules to calculate the chance value at each sensor nodes. Thus this technique is an only further extension of LEACH protocol and also possess same defects as exists in LEACH.

In [7] authors proposed a fuzzy approach for energy optimized routing. In this technique, all decision regarding cluster head selection is taken at the gateway. The issue of cluster formation is not considered in this technique. In [8] authors highlighted the limitation of LEACH [1] and further improved the performance of LEACH by using fuzzy logic and presented centralized clustering technique LEACH-FL. This technique is similar to the [5] approach except for input variables. LEACH-FL possess same limitations as Gupta's technique. In [9] authors have been presented multi-hop, centralized clustering technique CFGA (clustering WSN using fuzzy logic and genetic algorithm). The output of the fuzzy logic system with input variables: distance, energy, and density are used to set the value of timer at each sensor node. All sensor nodes start the countdown at the same time and nodes whose timer reaches zero defines itself as a cluster head candidate the BS. Then, the BS selects suitable cluster heads from the set of cluster head candidates by using optimization algorithm known as a genetic algorithm.

In [10] authors have been proposed clustering routing protocol based on fuzzy inference for WSNs (CEFM). This is localized clustering technique, where the decision regarding cluster heads selection depends on upon fuzzy system output. In CEFM new fuzzy input variable data retransmission rate is used with other input variables. In this technique, authors have not given any method to find out data retransmission rate. In [11] authors have been presented a cluster head election algorithm for WSN using Takagi-Sugeno fuzzy system (CHEATS). In CHEATS algorithm Takagi-Sugeno fuzzy inference system is used to compute the probability to become cluster heads. Two input variables: remaining energy and distance of a node from the base station are used to evaluate probability to become cluster head. CHEATS shows $10 \%$ improvement in network lifetime and $50 \%$ in throughput over LEACH protocol.

In [12] authors have been introduced heterogeneity in the network and presented cluster head election scheme based on the fuzzy system. Three input variables: selection probability, distance from the base station and the sum of distances from the all neighboring nodes are used to determine the chance value of nodes to become cluster head.

In [13] authors proposed a clustering approach which is similar to Gupta approach with two fuzzy input variables. Authors in [14] presented type-2 Takagi-Sugeno-Kang fuzzy logic system in clustering algorithm (ICT2TSK). This is centralized clustering algorithm, where the BS calculates the probability of each node to become a cluster head and also used optimum cluster radius to make cluster heads more uniform and balanced. In literature, most of the clustering techniques have not covered inter-cluster routing methods, so to keep in mind inter-cluster routing in [15] authors have been presented improved fuzzy unequal clustering (IFUC) scheme based on Ant Colony Optimization (ACO) method.

In [16] authors investigated and highlighted the limitations of LEACH [1], LEACH-C [10] and CHEF [13] techniques and further presented fuzzy logic based clustering technique LEACH-ERE. The output chance value is evaluated using two fuzzy input variables: expected residual energy (ERE) and residual energy of sensor nodes. Energy prediction is first time used in LEACH-ERE for selecting suitable sensor node as a cluster head. The expected residual energy can be predicted by using the offline trained neural network. Most of the clustering techniques have not considered the consequences of cluster head failure, because if cluster head fails due to lack of energy or physical damage then transferred data will be lost. Thus the idea of backup cluster head is introduced by the in [17] and presented self-configured $\mathrm{CH}$ selection ( $\mathrm{SCCH}$ ) technique using the type-2 fuzzy logic system to draw the inference from linguistic inputs. In SCCH after forming clusters, each cluster head selects backup cluster head with the highest chance value among cluster members. When cluster head loses its energy below a threshold level, it declares backup cluster head as a new cluster head and if backup cluster head also fails, then sensor nodes select other nodes as cluster head with highest chance value. In [18] again authors have presented backup 
cluster head concept with an improved algorithm using the type-2 fuzzy logic system. In [19] authors deployed sensors with different battery power and improved network lifetime by exploiting heterogeneity. The whole network is divided into circular disks based on the distance from the BS and further disks are segregated into clusters in [20].

In [21] authors done analysis on input parameters to anticipate the effect of different parameters on the lifetime of network using fuzzy logic. None of above approaches exploited the spatial correlation between sensors. Therefore, to exploit correlation authors have been formulated the novel correlation model and studied the impact in [22]. The distance calculation based on received signal strength is explained in [23]. In [24] authors comprehended the detailed review of existing fuzzy based clustering techniques. In order to overcome the problem of uncertainty in cluster head selection and implementation of spatial correlation at the network layer, this paper presents a novel method to segregate the network uniformly with the help of fuzzy logic and spatial correlation model. In next section, the problem is formulated.

\section{PROBLEM FORMULATION}

Let $N$ number of sensors $\left(s_{i}\right)$ deployed in the area of interest (AoI). The sensors are equally capable and can sense in omnidirectional within its sensing radius. Fig. 2 illustrate the overlapped sensing region of various sensors. The common sensing region depends upon the placement of the sensors in the field.

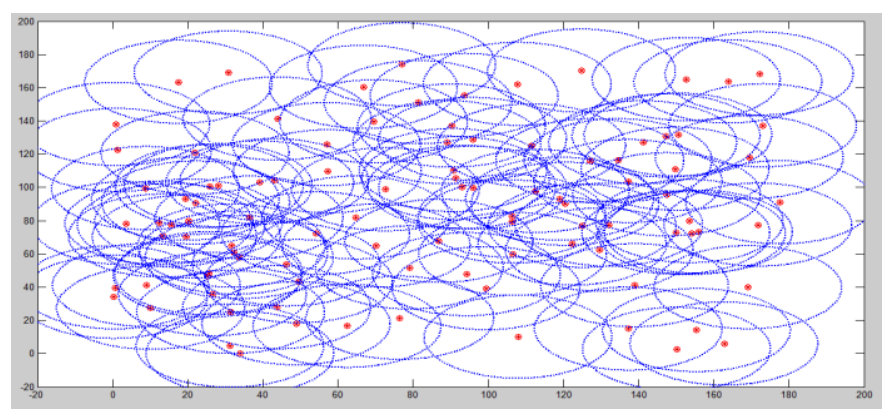

Fig. 2. Overlapped sensing region in WSN.

Now in clustering process, if cluster framed in such a way that all the cluster members are closely located to each other. Then cluster members have to send data within less distance. Therefore, energy expenditure at the transceiver system can be reduced because most of the energy expended in transmitting and receiving the data [1]. In clustering, the main problem is to identify the most efficient cluster heads in the network as shown in Fig. 1 and then add members to the cluster whose location spatially close to each other. Therefore keeping in mind these problems a generic fuzzy based clustering approach is developed in next section.

\section{Proposed Clustering APPROACH}

The proposed approach configure clusters in two phases similar to the LEACH [1] and CHEF [6]. It is assumed that the geographical location of sensor nodes is identified by using any of localization technique. In initial setup after deployment of sensors in the AoI, the base station (BS) broadcast the 'START' message in the network. The sensor nodes on receiving the message evaluate the distance between them according to the signal strength as follows [23]:

$$
d=10^{\wedge}\left[\left(P_{o}-F_{m}-P_{r}-10 L \log _{10}(f)+30 L-32.44\right) / 10 L\right.
$$

The meaning of symbols used in (1) are given in Table 2. Now each sensor node approximates the required energy to transmit $b$ bits to the BS as follows [1]:

$$
E_{T x}(b, d)=\left\{\begin{array}{ll}
\left(E_{\text {elec }}+\varepsilon_{f s} \times d^{2}\right) \times b, & d<d_{0} \\
\left(E_{\text {elec }}+\varepsilon_{m p} \times d^{4}\right) \times b, & d \geq d_{0}
\end{array}\right\}
$$

The meaning of symbols used in (2) are given in Table 2. Now every sensor node in the field knows required energy to transmit $b$ bits to the BS. In next step each sensor node calculates the probability to become a cluster head $(P)$ based on residual energy as follows:

$$
s(i) \cdot \text { probability }=E_{r} / E_{0}
$$

Where $E_{r}$ is the residual energy and $E_{0}$ is the initial energy value. Therefore, according to (3) initially, all sensor nodes are equally probable to become a cluster head but with an increase in a number of rounds energy expenditure increase and each sensor node possess the different probability of becoming a cluster head. The sensor nodes are embedded with a fuzzy system [5], [6]. Now each sensor node use required energy to transmit and probability as fuzzy system input variable and evaluate the chance to become a cluster head.

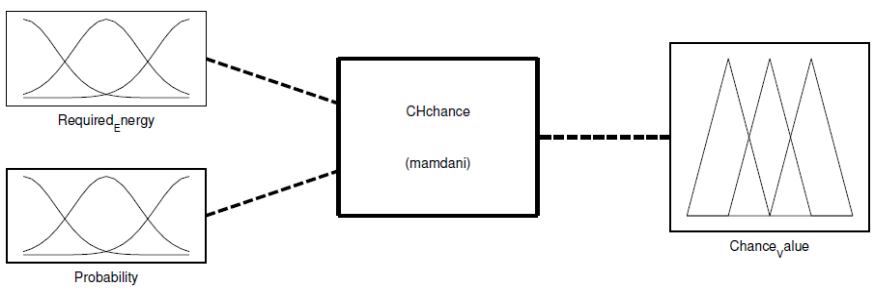

Fig. 3. Fuzzy model to evaluate output chance value.

The fuzzy system is shown in Fig. 3 and fuzzy if-then rules are listed in Table 1. The membership functions for inputs and output are depicted in Fig. 4, 5 and 6, respectively.

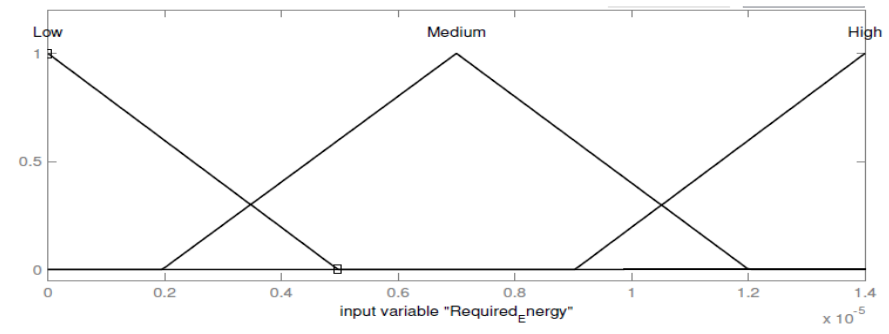

Fig. 4. Membership function for input variable (required energy).

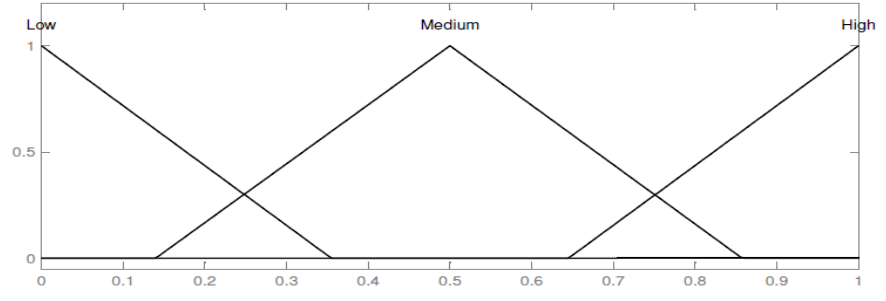

Fig. 5. Membership function for input variable (Probability). 


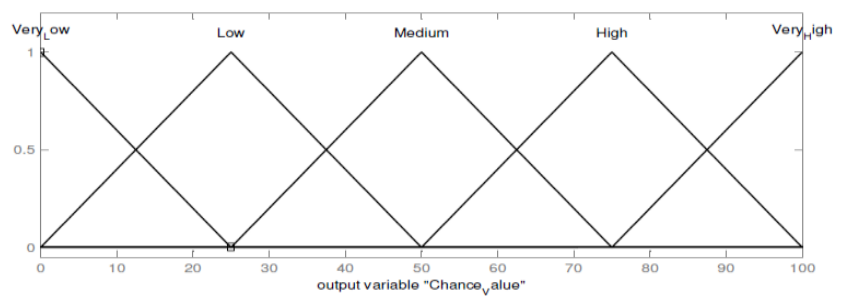

Fig. 6. Membership function for output variable (chance value).

TABLE I. FUZZY IF-THEN RULES

\begin{tabular}{|l|l|l|l|}
\hline Rule No. & Required Energy & Probability & Chance Value \\
\hline 1 & Low & Very low & Low \\
\hline 2 & Low & Low & Low \\
\hline 3 & Low & Medium & High \\
\hline 4 & Low & High & Very high \\
\hline 5 & Medium & Very low & Very low \\
\hline 6 & Medium & Low & Very low \\
\hline 7 & Medium & Medium & Medium \\
\hline 8 & Medium & High & Very high \\
\hline 9 & High & Very low & Very low \\
\hline 10 & High & Low & Very low \\
\hline 11 & High & Medium & Low \\
\hline 12 & High & High & Medium \\
\hline
\end{tabular}

The range for required energy is [0 .000014], which is calculated according to (2) for the farthest sensor node in the field of area $150 \times 150 \mathrm{~m}^{2}$. The fuzzy system gives crisp output value. Now every sensor node set its back off time as follows:

$$
s(i) \cdot \text { backoff }=\frac{1}{s(i) \cdot \text { chance }} \mu \mathrm{sec}
$$

According to the applied fuzzy if-then rules and (4) backoff time is lower for the more eligible sensor node compare to the less eligible sensor node. The sensor nodes broadcast itself as cluster head candidates within the optimal cluster radius after waiting for backoff time. The optimal cluster radius is equal to the twice of the sensing range of the sensor. If two sensor node at the same time declares itself as a cluster head candidate then the sensor node with higher chance value become the cluster head for the current round and other nodes join as a cluster member. During the backoff time, other sensors receive cluster head candidate message.

The sensor node checks the correlation value between the cluster head candidate and itself. If the correlation value is higher than user-defined correlation threshold value then sends the cluster join message to the cluster head candidate. However, if more than one cluster head candidate messages received in such scenario sensor node decide on the basis of the correlation value. Thus sensor node joins the cluster candidate with higher correlation value between them. The correlation value between sensors is evaluated according to the spatial correlation model given in [22] as follows:

$$
\rho_{(i, j)}=\left(\frac{2 v^{2} \cos ^{-1}\left(d_{(i, j)} / v\right)-2 d_{(i, j)} \sqrt{\left(v^{2}-d_{(i, j)}^{2}\right)}}{\pi v^{2}}, \text { if } \quad 0 \leq d_{(i, j)} \leq v\right.
$$

The meaning of symbols used in (5) are listed in Table 2. The correlation value matrix is evaluated at initial step by the BS using (5) and broadcasted in the network. Therefore each sensor node knows the correlation value with respect to every sensor node in the network. In this proposed scheme the correlation threshold value is equal to 0.5 . It means the sensor nodes whose correlation value is greater than the 0.5 are considered as the correlated to each other. Therefore the cluster heads are elected based on the fuzzy output chance value and cluster formation is done on the basis of the correlation value between them. The algorithm for proposed approach is given in Algorithm 1.

TABLE II. LIST OF NOTATIONS AND SYMBOLS

\begin{tabular}{|l|l|}
\hline Notations/symbols & Meaning \\
\hline$s(i)$ & Sensor node \\
\hline$P_{r}$ & Power received \\
\hline$E$ & Residual energy \\
\hline$R$ & Sensing radius of sensor \\
\hline$d_{(i, j)}$ & Distance between two nodes $(s(i)$ and $s(j))$ \\
\hline$d$ & Actual distance between node and sink \\
\hline$d_{0}$ & Threshold distance \\
\hline$P_{0}$ & Power received at $d_{0}$ \\
\hline$L$ & Path loss exponent \\
\hline$f$ & Frequency \\
\hline$\varepsilon_{f s}$ & Free space amplification factor \\
\hline$\varepsilon_{m p}$ & Multipath amplification factor \\
\hline$b$ & Number of bits in a packet \\
\hline$E_{T x}(b, d)$ & Required energy to transmit $b$ bits to distance $d$ \\
\hline$E_{e l e c}$ & Energy consumed by electrical circuit \\
\hline$E_{D A}$ & Energy expenditure for data aggregation \\
\hline$v$ & Control parameter $(v=2 R)$ \\
\hline$\rho_{(i, j)}$ & Correlation value between $(s(i)$ and $s(j))$ \\
\hline
\end{tabular}

Algorithm 1. Proposed Clustering Approach

Function: proposed ( $N$, required energy, probability)

Round $\leftarrow 1$;

Initialization:

The base station broadcast 'START' message;

Calculate correlation matrix using Eq. (5);

Calculate required energy to transmit $b$ bits (Eqs. (1) \& (2));

Start

1. For $\mathrm{i}=1: \mathrm{N}$

2. fis $=$ readfis ('CHchance');

3. $s(i) \cdot$ chance $=$ evalfis([s(i).probability s(i).required-energy], fis);

4. $\quad \mathrm{s}(\mathrm{i}) \cdot$ backoff $=1 / \mathrm{s}(\mathrm{i})$. chance;

5. Countdown begin

6. Declare itself as cluster head candidate after waiting for back off time;

7. Elseif cluster head candidate messages received during back off time;

8. Check correlation value w.r.t received cluster head candidates;

9. If anyone has correlation $>0.5$

10. Stop waiting;

11. Join cluster head having highest value of correlation;

12. Else

13. Declare itself the cluster head candidate after back off time;

14. Wait for cluster head join messages from other nodes;

15. Cluster head design TDMA schedule for cluster members;

16. End

17. End

18. End

19. Cluster head send aggregated data to the BS;

20. Calculate energy expended in current round;

21. Check for alive nodes

22. If alive nodes $>0$

23. Round $\leftarrow$ Round +1 ;

24. Go to step 1;

25. End 


\section{RESUlTS AND DisCUSSIONS}

In this section, the performance of proposed approach is evaluated using MATLAB simulations. The network lifetime is defined on the basis of the metrics such as the first node die round (FND), the last node die round (LND), alive node in each round and average energy consumption per round. The WSN is created in the simulator for random deployment of 100 and 200 nodes respectively in the field dimension $150 \times$ $150 \mathrm{~m}^{2}$. The BS is located outside the field at $(75,175)$. The simulation parameter used are listed in Table 3. The cluster formation in the network using proposed approach for random distribution of 100 and 200 nodes are shown in Fig. 7 and 8, respectively.

It is clearly seen from the results that the clusters are uniformly distributed throughout the network and cluster members are spatially close to each other in the field. The comparison for alive nodes per round is depicted in Fig. 9 and 10 for 100 and 200 nodes in the network. It is observed from results that the first node die round is later for proposed approach in 100 and 200 nodes random deployment as compared to the LEACH and CHEF. When any node dies due to depletion of battery power than the region covered by node get disconnected from rest of the network. Therefore the network is considered as dead so when the first node dies in later round the network lifetime is better. The FND and LND comparison are given in Fig. 11 and 12, respectively. It is inferred that proposed approach perform better in term of FND and LND as compared to the LEACH and CHEF.

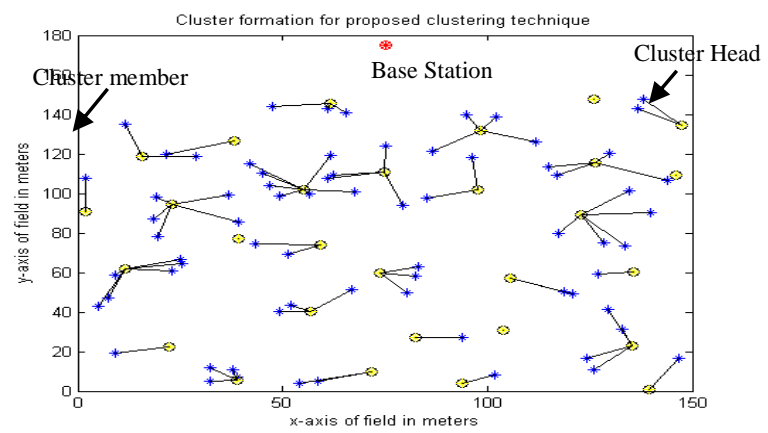

Fig. 7. Cluster formation in the network for 100 nodes.

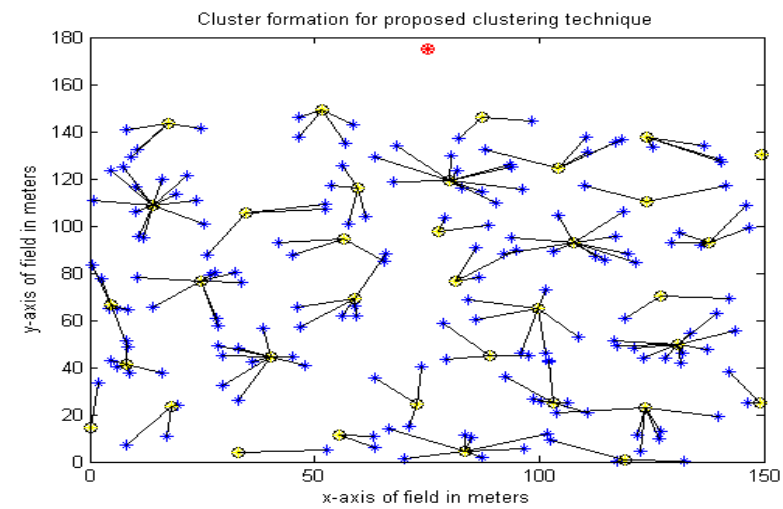

Fig. 8. Cluster formation in the network for 200 nodes.

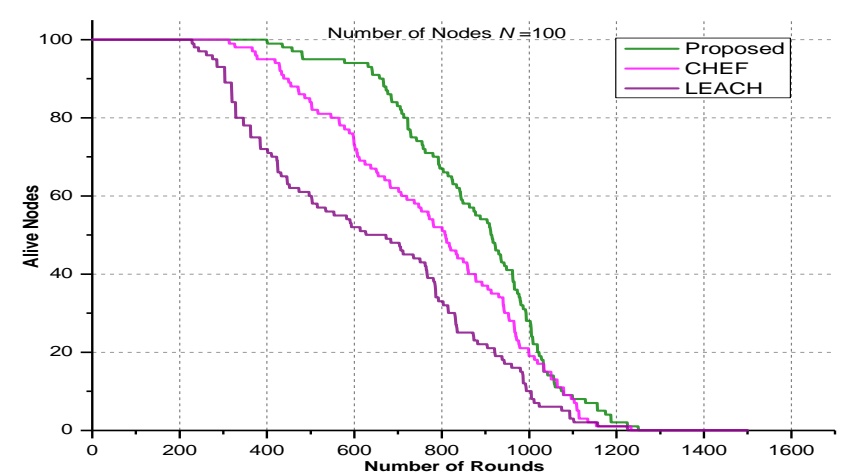

Fig. 9. Alive nodes per round for random deployment of 100 nodes.

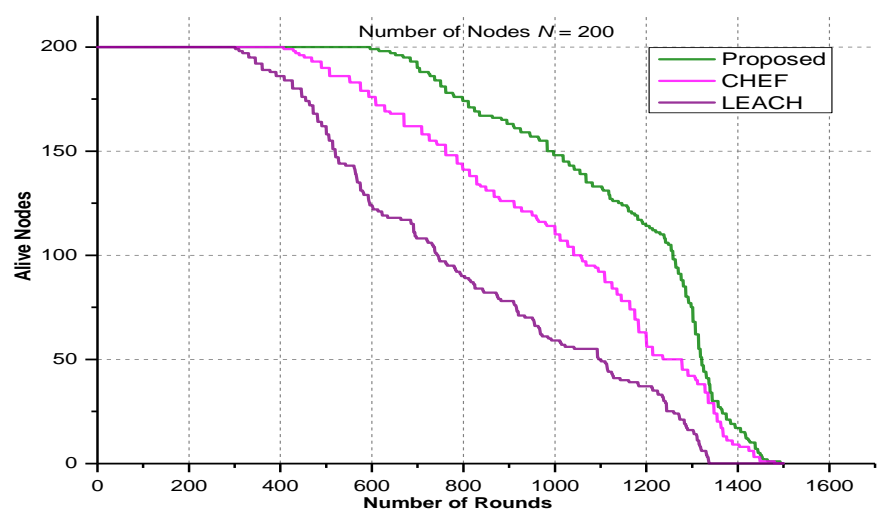

Fig. 10. Alive nodes per round for random deployment of 200 nodes.

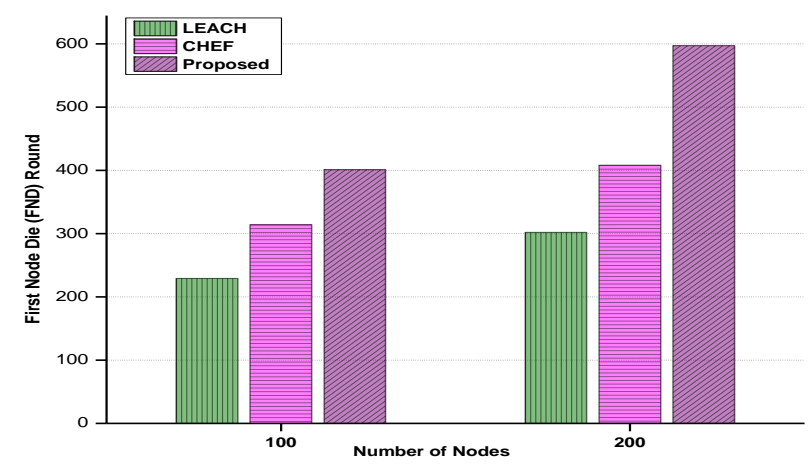

Fig. 11. FND comparison.

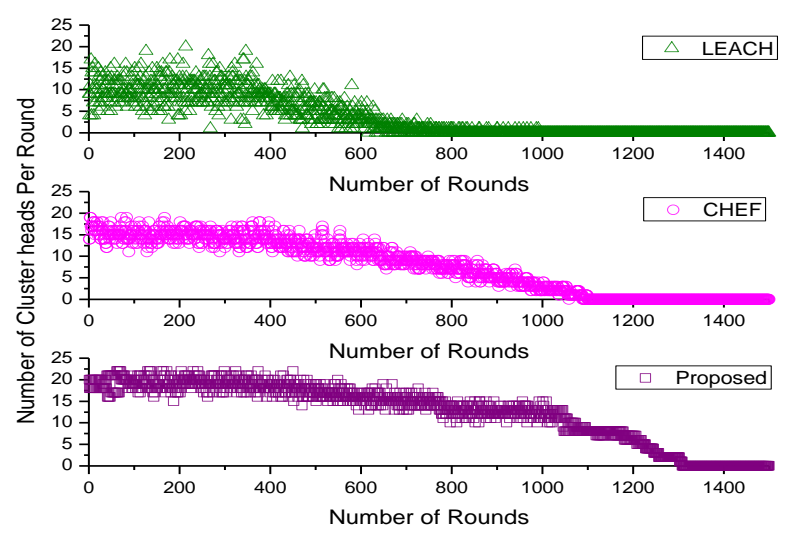

Fig. 12. Cluster heads distribution per round. 
TABLE III. SIMULATION PARAMETERS

\begin{tabular}{|l|l|}
\hline Parameter & Value \\
\hline 2D Field dimensions & $150 \times 150 \mathrm{~m}^{2}$ \\
\hline Sensors and the BS & Static \\
\hline The base station position & $(\mathrm{x}=75, \mathrm{y}=175)$ \\
\hline Initial energy $\left(E_{0}\right)$ & $1 \mathrm{Joule}$ \\
\hline$\varepsilon_{f s}$ & $10 \times 10^{-12} \mathrm{~J} / \mathrm{bit}^{\mathrm{s}} \mathrm{m}^{2}$ \\
\hline$\varepsilon_{m p}$ & $.0013 \times 10^{-12} \mathrm{~J} / \mathrm{bit} / \mathrm{m}^{4}$ \\
\hline Number of Nodes $(N)$ & 100,200 \\
\hline$E_{\text {elec }}$ & $50 \mathrm{~nJ}$ \\
\hline$E_{D A}$ & $5 \mathrm{~nJ}$ \\
\hline Fuzzy rules & 12 \\
\hline Node distribution & Random \\
\hline header length & 25 bytes \\
\hline Broadcast packet length & 16 bytes \\
\hline Packet length & 600 bytes \\
\hline Optimal cluster radius & $2 \mathrm{R}=20 \mathrm{~m}$ \\
\hline Number of BS & 1 \\
\hline Correlation threshold value & 0.5 \\
\hline
\end{tabular}

The cluster heads distribution is given in Fig. 13 for random deployment of 100 nodes. The cluster heads distribution in proposed approach is uniform like CHEF [6] approach due to the optimal cluster radius whereas in $\mathrm{LEACH}$ [1] the distribution is random. Therefore uniform cluster distribution is achieved by using proposed approach.

The average energy consumption for a random distribution of 100 nodes is illustrated in Fig. 14 where each value is the average of 100 simulations respective to the different rounds up to first 1000 rounds. It can be seen from the result that energy consumption for proposed approach is less as compared to the LEACH [1] and CHEF [6]. Therefore after evaluating the performance of proposed approach with respect to the different metrics, it can infer that proposed approach is more energy efficient and able to extend the network lifetime as compared to the LEACH [1] and CHEF [6], respectively.

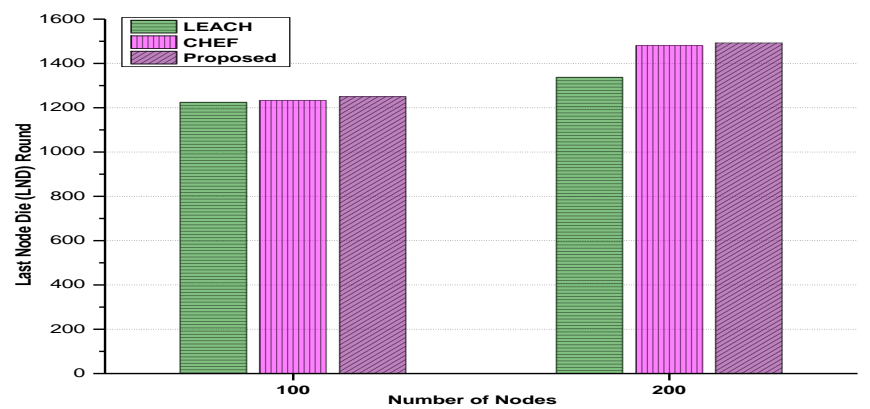

Fig. 13. LND comparison.

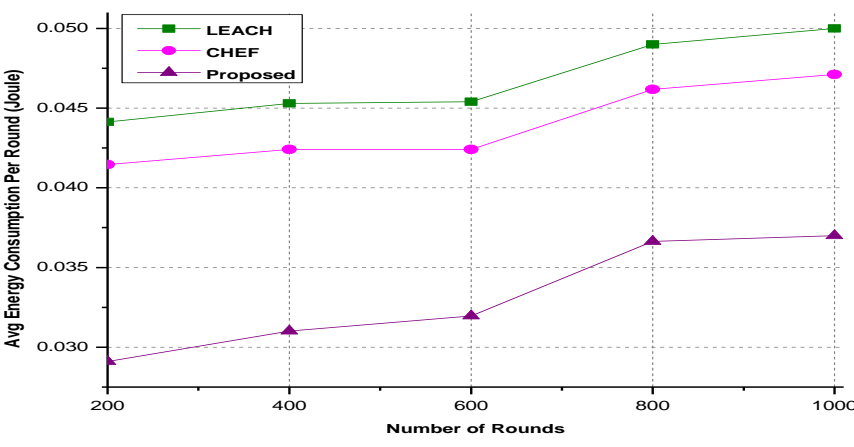

Fig. 14. Average energy consumption for first 1000 rounds.

\section{CONCLUSION}

In this paper, a basic fuzzy based clustering approach has been introduced. Using proposed approach various network lifetime key elements is discussed. It is found that correlation characteristics can be used to organize the clusters between spatially closed sensor nodes and the cluster heads can be identified on the basis of the fuzzy system output value. The results show that for the proposed approach average energy consumption is low and perform better in term of FND, LND, cluster heads distribution and alive nodes per round. Therefore, proposed approach enhances the network operational lifetime. In addition, a comparative study showed that the proposed approach outperforms LEACH and $\mathrm{CHEF}$ techniques. As a future work, the more realistic correlation can be developed for cluster formation based on a fuzzy logic system with accounting other membership functions.

\section{REFERENCES}

[1] W. Heinzelman, A. Chandrakasan and H. Balakrishnan, "An application-specific protocol architecture for wireless microsensor networks", IEEE Transactions on Wireless Communications, vol. 1, no. 4, pp. 660-670, 2002.

[2] O. Younis and S. Fahmy, "HEED: a hybrid, energy-efficient, distributed clustering approach for ad hoc sensor networks", IEEE Transactions on Mobile Computing, vol. 3, no. 4, pp. 366-379, 2004.

[3] S. Shi, X. Liu and X. Gu, "An energy-efficiency Optimized LEACH-C for wireless sensor networks", Proceedings of the 7th ICST International Conference on Communications and Networking in China (CHINACOM), 2012, pp. 487-492.

[4] F.A. Aderohunmu, J.D. Deng and M.K. Purvis, "A deterministic energyefficient clustering protocol for wireless sensor networks", Proceedings of the 7th IEEE International Conference on Intelligent Sensors, Sensor Networks and Information Processing (ISSNIP), 2011, pp. 341-346.

[5] Gupta, D. Riordan and S. Sampalli, "Cluster-head election using fuzzy logic for wireless sensor networks". Proceedings of the 3rd IEEE Annual Conference on Communication Networks and Services Research, 2005, pp. 255-260.

[6] J.M. Kim, S.H. Park, Y.J. Han and T.M. Chung, "CHEF: cluster head election mechanism using fuzzy logic in wireless sensor networks", Proceedings of the 10th IEEE International Conference on Advanced Communication Technology (ICACT), 2008, 654-659.

[7] T. Haider and M. Yusuf, "A fuzzy approach to energy optimized routing for wireless sensor networks", Int. Arab J. Inf. Technol. Vol. 6, No. 2, 2009, pp. 179-185.

[8] G. Ran, H. Zhang and S. Gong, "Improving on LEACH Protocol of Wireless Sensor Networks Using Fuzzy Logic", Journal of Information and Computational Science Vol. 7, No. 3, 2010, pp. 767-775.

[9] E. Saeedian, M.N. Torshiz, M. Jalali, G. Tadayon and M.M. Tajari, "CFGA: Clustering wireless sensor network using fuzzy logic and genetic algorithm", Proceedings of the 7th IEEE International 
Conference on Wireless Communications, Networking and Mobile Computing (WiCOM), 2011, pp. 1-4.

[10] R. Jin, N. Wei, X. Shi, T. Gao and J. Zou, "Clustering routing protocol based on fuzzy inference for WSNs", Proceedings of the 7th IEEE International Conference on Wireless Communications, Networking and Mobile Computing (WiCOM), 2011, pp. 1-4.

[11] Pires, C. Silva, E. Cerqueira, D. Monteiro and R. Viegas, "CHEATS: A cluster-head election algorithm for WSN using a Takagi-Sugeno fuzzy system", Proceedings of the IEEE Latin-American Conference on Communications (LATINCOM), 2011, pp. 1-6.

[12] Y. Shen and H. Ju, "Energy-Efficient Cluster-Head Selection based on a Fuzzy Expert System in Wireless Sensor Networks", Proceedings of the IEEE/ACM International Conference on Green Computing and Communications, 2011, pp. 110-113.

[13] Z.W. Siew, A. Kiring, H.T. Yew, P. Neelakantan, and K.T.K. Teo, "Energy Efficient Clustering Algorithm in Wireless Sensor Networks Using Fuzzy Logic Control", Proceedings of the IEEE Colloquium on Humanities, Science and Engineering Research (CHUSER), 2011, pp. 392-397.

[14] F. Zhang, Q. Zhang and Z. Sun, "ICT2TSK: An improved clustering algorithm for WSN using a type-2 Takagi-Sugeno-Kang Fuzzy Logic System", Proceedings of the IEEE Symposium on Wireless Technology and Applications (ISWTA), 2013, pp. 153-158.

[15] S. Mao, C. Zhao, Z. Zhou and Y. Ye, "An Improved Fuzzy Unequal Clustering Algorithm for Wireless Sensor Network", Mobile Networks and Applications, Vol. 18, No. 2, 2012, pp. 206-214.
[16] J. Lee and W. Cheng, "Fuzzy-Logic-Based Clustering Approach for Wireless Sensor Networks Using Energy Predication", IEEE Sensors J. Vol. 12, No. 9, 2012, pp. 2891-2897.

[17] D. Izadi, J. Abawajy and S. Ghanavati, "A new energy efficient clusterhead and backup selection scheme in WSN", Proceedings of the 14th IEEE International Conference on Information Reuse \& Integration (IRI), 2013, pp. 408-415.

[18] D. Izadi, J. Abawajy and S. Ghanavati, "An Alternative Clustering Scheme in WSN", IEEE Sensors J. Vol. 15, No. 7, 2015, pp. 4148-4155.

[19] Devasena and B. Sowmya. "Fuzzy Based BEENISH Protocol for Wireless Sensor Network", Circuits and Systems, Vol. 7, No. 8, 2016, pp. 1893.

[20] M. Tamene and K.N. Rao, "Fuzzy Based Distributed Cluster Formation and Route Construction in Wireless Sensor Networks", International Journal of Computer Applications, Vol. 140, No. 5, 2016, pp. 21-27.

[21] Q. Wang, E. Kulla, G. Mino and L. Barolli, "Prediction of Sensor Lifetime in Wireless Sensor Networks Using Fuzzy Logic", Proceedings of the 28th IEEE International Conference on Advanced Information Networking and Applications, 2014, pp. 1127-1131.

[22] R.K. Shakya, Y.N. Singh and N.K Verma, "Generic correlation model for wireless sensor network applications", IET Wireless Sensor Systems, Vol. 3, No. 4, 2013, pp. 266-276.

[23] J. Xu, W. Liu, F. Lang, Y. Zhang and C. Wang, "Distance measurement model based on RSSI in WSN", Wireless Sensor Network, Vol. 2, No. 8, 2010, pp. 606-11.

[24] M. Singh, S. K. Soni, "A comprehensive review of fuzzy-based clustering techniques in wireless sensor networks," Sensor Review, Vol. 37, No. 3, June 2017, pp.289-304. 